2. Eisenberg T, Fawzy A, Nicklas W, Semmler T, Ewers C. Phylogenetic and comparative genomics of the family Leptotrichiaceae and introduction of a novel fingerprinting MLVA for Streptobacillus moniliformis. BMC Genomics. 2016;17:864. http://dx.doi.org/10.1186/s12864-016-3206-0

3. Dendle C, Woolley IJ, Korman TM. Rat-bite fever septic arthritis: illustrative case and literature review. Eur J Clin Microbiol Infect Dis. 2006;25:791-7. http://dx.doi.org/10.1007/s10096-006-0224-x

4. Gilroy SA, Khan MU. Rat bite fever: case report and review of the literature. Infectious Diseases in Clinical Practice. 2002;11:403-5. http://dx.doi.org/10.1097/00019048-200209000-00007

5. Irvine L, Wills T. Streptobacillus moniliformis: a mouse trying to become a rat. Clinical Microbiology Newsletter. 2006;28:118-20. http://dx.doi.org/10.1016/j.clinmicnews.2006.07.002

6. Trigo-Daporta M, Cortizo-Vidal S, Pallarés-González Á, García-Campello M. Fever and rash in the owner of exotic pets [in Spanish]. Enferm Infecc Microbiol Clin. 2011;29:311-2. http://dx.doi.org/10.1016/j.eimc.2010.07.019

Address for correspondence: Antoine Guillon, Centre Hospitalier Regional Universitaire de Tours, 2 Blvd Tonnelle, Tours 37044, France; email: antoine.guillon@univ-tours.fr

\section{Malaria in Children Adopted from the Democratic Republic of the Congo}

\section{Elena Chiappini, Sara Sollai, Maurizio de Martino, Luisa Galli}

Author affiliation: Meyer University Hospital, Florence University, Florence, Italy

DOI: http://dx.doi.org/10.3201/eid2304.161777

Data are lacking regarding asymptomatic and symptomatic malaria prevalence in internationally adopted children. Among 20 children from Democratic Republic of the Congo evaluated in Florence, Italy, in April 2016, malaria prevalence was $80 \% ; 50 \%$ of infected children had symptomatic malaria. Adopted children from areas of high malaria endemicity should be screened for malaria.

$\mathrm{T}$ he Democratic Republic of the Congo (DRC) banned adoption of children by parents from other countries in 2013. In February 2016, the ban was removed, and several hundred children were allowed to join families in Europe and in the United States. The first group of children from DRC arrived in Italy in April 2016, and 20 children were referred to the Center for Internationally Adopted Children at Meyer University Hospital, Florence, Italy. All children underwent the standard infectious disease screening tests recommended by the American Academy of Pediatrics, including those for tuberculosis (by tuberculin skin test and interferon- $\gamma$ release assay) and intestinal parasites (by fecal testing for ova, parasites, and antigen test for Giardia spp.) and serologic tests for Toxocara canis, Strongyloides spp., hepatitis $\mathrm{B}$ and $\mathrm{C}$ viruses, HIV-1/2 viruses, and Treponema pallidum (syphilis) (1).

Eight children who were exhibiting fever were admitted to the hospital and received a diagnosis of malaria soon after their arrival in Italy. For malaria testing, PCR and microscopy were performed on thin and thick smears. Parasitemia level was determined by counting the parasitized erythrocytes among the 500-2,000 erythrocytes on the thin smear and calculating the percentage.

The remaining children from DRC were screened, and another 8 children were found to be infected. Thus, malaria was diagnosed in 16 children ( 10 were boys; median age 7 years [range $4-10$ years]), and malaria prevalence was $80 \%$ (16/20). Plasmodium falciparum infection was documented in 15 cases, whereas a mixed infection (P. falciparum and $P$. ovale) was observed in 1 child. All children underwent treatment with intravenous quinine plus artesunate or oral dihydroartemisinin/piperaquine (2). Intravenous treatment was administered to 1 child who had severe malaria (generalized seizures) and to 5 children with a parasitemia level $\geq 2 \%$ or who exhibited vomiting and therefore were unable to take oral medications reliably (2). Because intravenous artesunate is unlicensed in Europe but is available in our center, we obtained written informed consent for its use from the patients' parents before administration. The study received approval by Meyer University Hospital Ethics Committee.

Other studies have assessed the prevalence of malaria in internationally adopted children. Among a population of 182 children in France, when Blanchi et al. screened for the children originating from malaria-endemic zones who were exhibiting fever, splenomegaly, or both for malaria, they found 2 infected children (3). More recently, Adebo et al. screened 52 children arriving in the United States from Ethiopia for malaria and reported 7 (13.5\%) children with asymptomatic malaria (4). These authors suggested that screening be conducted of children coming from malariaendemic areas with noted risk factors, such as splenomegaly (4). Anemia (hemoglobin levels $<11 \mathrm{~g} / \mathrm{dL}$ ) could be another risk factor for malaria but, because it is common in this population, it was not considered in the study by Adebo et al. (4). However, in our population, we found that tests for parasitemia were positive for 6 children without splenomegaly or hepatomegaly and for 1 child who had neither anemia $(<11 \mathrm{~g} / \mathrm{dL})$, hepatomegaly, nor splenomegaly. Therefore, using clinical features to select children who should undergo the screening may be challenging. 
Given the paucity of literature data regarding malaria prevalence in internationally adopted children, testing by PCR, microscopy, or both, followed by treatment of infected children, would be preferable to the empiric treatment, considering the costs and possible adverse effects of antimarial drugs. Moreover, the preferable screening strategy is not apparent. We did not observe any discrepancy between microscopy and PCR results; however, a higher sensitivity by PCR has been reported $(4,5)$. In contrast, some experts prefer testing by microscopy examination because PCR techniques are not sufficiently standardized or validated to be used for routine clinical diagnosis (2).

In our dataset, malaria prevalence was substantially higher than that previously reported (4). This finding may be due to the particular situation of these children and to orphanage conditions (i.e., lack of mosquito nets). Moreover, it should be noted that, to date, 3 countries - DRC, Nigeria, and India - account for $40 \%$ of all estimated malaria cases in the world (6). Also, a high prevalence of asymptomatic malaria in DRC has been reported, in $\approx 15 \%$ of children $(7,8)$.

Our results should be interpreted with caution, given the small dataset, but they should alert pediatricians regarding the importance of assessing malaria risk in children who have been adopted internationally. The degree of malaria endemicity in the child's area of origin may be considered in the decision to screen asymptomatic children adopted in non-malaria-endemic countries. In particular, children who come from areas of high malaria endemicity, such as DRC, deserve a careful screening, even in the absence of any sign or symptom.

Dr. Chiappini is an associate professor of pediatrics at the University of Florence. She is also the head of the unit for internationally adopted children at Meyer University Hospital in Florence. Her research interests focus on pediatric infectious diseases, including HIV infection, tuberculosis, and malaria.

\section{References}

1. American Academy Pediatrics Committee on Infectious Diseases. Medical evaaluation of internationally adopted children for infectious disease. In: Pickering LK, Baker CJ, Kimberlin DW, editors. Red book: report of the Committee on Infectious Diseases. 29th ed. Elk Grove Village (IL): The Academy; 2012. p. 192-200.

2. Lalloo DG, Shingadia D, Bell DJ, Beeching NJ, Whitty CJ, Chiodini PL; PHE Advisory Committee on Malaria Prevention in UK Travellers. UK malaria treatment guidelines 2016. J Infect. 2016;72:635-49. http://dx.doi.org/10.1016/j.jinf.2016.02.001

3. Blanchi S, Chabasse D, Pichard E, Darviot E, de Gentile L. Post-international adoption medical follow-up at the Angers university hospital between 2009 and 2012. Med Mal Infect. 2014;44:69-75. http://dx.doi.org/10.1016/j.medmal.2013.12.003

4. Adebo SM, Eckerle JK, Andrews ME, Howard CR, John CC. Asymptomatic malaria and other infections in children adopted from Ethiopia, United States, 2006-2011. Emerg Infect Dis. 2015;21:1227-9. http://dx.doi.org/10.3201/eid2107.141933

5. Menge DM, Ernst KC, Vulule JM, Zimmerman PA, Guo H, John CC. Microscopy underestimates the frequency of Plasmodium falciparum infection in symptomatic individuals in a low transmission highland area. Am J Trop Med Hyg. 2008;79:173-7.

6. World Health Organization. WHO malaria report 2012 [cited 2016 Oct 31]. https://www.k4health.org/sites/default/files/who_malaria_ report_2012.pdf

7. Maketa V, Mavoko HM, da Luz RI, Zanga J, Lubiba J, Kalonji A, et al. The relationship between Plasmodium infection, anaemia and nutritional status in asymptomatic children aged under five years living in stable transmission zones in Kinshasa, Democratic Republic of Congo. Malar J. 2015;14:83. http://dx.doi.org/10.1186/ s12936-015-0595-5

8. Myumbi DM, Bobanga TL, Melin P, De Mol P, Kayembe JM, Situakibanza HN, et al. The prevalence of Plasmodium falciparum infection in asymptomatic individuals from the Democratic Republic of Congo. Malar Res Treat. 2016; 2016:5405802.

Address for correspondence: Elena Chiappini, Infectious Disease Unit, Meyer University Hospital, Department of Health Science, Florence University, Viale Pieraccini, 24, Florence, Italy; email: elena.chiappini@unifi.it

\section{LETTER}

\section{Cord Blood Sample Screening for Evidence of Maternal Chagas Disease}

\section{Susan P. Montgomery, Susan L. Stramer}

Author affiliations: Centers for Disease Control and Prevention, Atlanta, Georgia, USA (S.P. Montgomery); American Red Cross, Gaithersburg, Maryland, USA (S.L. Stramer)

DOI: http://dx.doi.org/10.3201/eid2304.161287

To the Editor: The article by Edwards et al. (1) contained several errors regarding testing, results, and interpretation of results. The authors incorrectly described the testing performed for the cord blood samples. The American Red Cross (ARC) National Testing Laboratory (NTL) (identified as the "American Red Cross National Donor Testing Laboratory" in the article) has never performed indirect hemagglutination assay testing, a method not licensed by the Food and Drug Administration (FDA) for detection of antibodies to Trypanosoma cruzi. In fact, the laboratory used a combination of testing algorithms during 2007-2014, the period of the study, involving 2 different FDA-licensed screening tests and a combination of research and licensed supplemental tests. Each algorithm had varying positive predictive values, ranging from $<10 \%$ to $>50 \%$. The laboratory 